\title{
PICOT binding to chromatin-associated EED negatively regulates cyclin D2 expression by increasing H3K27me3 at the CCND2 gene promoter
}

\author{
Pinakin Pandya', Minesh Jethva', Eitan Rubin ${ }^{1}$, Ramon Y. Birnbaum², Alex Braiman and Noah Isakov (1)
}

\begin{abstract}
Protein kinase C (PKC)-interacting cousin of thioredoxin (PICOT; also termed glutaredoxin 3 (Grx3; Glrx3)) is a ubiquitous protein that can interact with the embryonic ectoderm development (EED) protein via each of its two Cterminal PICOT/Grx homology domains. Since EED is a Polycomb-Group protein and a core component of the polycomb repressive complex 2 (PRC2), we tested the involvement of PICOT in the regulation of PRC2-mediated H3 lysine 27 trimethylation (H3K27me3), transcription and translation of selected PRC2 target genes. A fraction of the cellular PICOT protein was found in the nuclei of leukemia cell lines, where it was associated with the chromatin. In addition, PICOT coimmunoprecipitated with chromatin-residing EED derived from Jurkat and COS-7 cell nuclei. PICOT knockdown led to a reduced H3K27me3 mark and a decrease in EED and EZH2 at the CCND2 gene promoter. In agreement, PICOT-deficient T cells exhibited a significant increase in CCND2 mRNA and protein expression. Since elevated expression levels of PICOT were reported in several different tumors and correlated in the current studies with decreased transcription and translation of the CCND2 gene, we tested whether this opposite correlation exists in human cancers. Data from the Cancer Genome Atlas (TCGA) database indicated statistically significant negative correlation between PICOT and CCND2 in eight different human tumors where the highest correlation was in lung $(p=8.67 \mathrm{E}-10)$ and pancreatic $(p=1.06 \mathrm{E}-5)$ adenocarcinoma. Furthermore, high expression of PICOT and low expression of CCND2 correlated with poor patient survival in five different types of human tumors. The results suggest that PICOT binding to chromatin-associated EED modulates the H3K27me3 level at the CCND2 gene promoter which may be one of the potential mechanisms for regulation of cyclin D2 expression in tumors. These findings also indicate that a low PICOT/CCND2 expression ratio might serve as a good predictor of patient survival in selected human cancers.
\end{abstract}

\section{Introduction}

Protein kinase $\mathrm{C}$ (PKC)-interacting cousin of thioredoxin (PICOT, also termed glutaredoxin 3 (Grx3; Glrx3)), was discovered in the year 2000 in a search for

\footnotetext{
Correspondence: Noah Isakov (noah@bgu.ac.il)

${ }^{1}$ The Shraga Segal Department of Microbiology, Immunology and Genetics, Faculty of Health Sciences and the Cancer Research Center, Ben Gurion University of the Negev, P.O.B. 653, 84105 Beer Sheva, Israel

${ }^{2}$ Department of Life Sciences, Ben-Gurion University of the Negev, 84105 BeerSheva, Israel

Edited by I. Amelio
}

PKC $\theta$ binding proteins ${ }^{1}$. PICOT possesses three evolutionary conserved domains including an N-terminal thioredoxin (Trx) homology domain and a tandem repeat of a PICOT/glutaredoxin (Grx) homology domain $^{2,3}$. However, in contrast to Trx and Grx, which possess two redox-active cysteines at their catalytic site, PICOT possesses only a single cysteine residue in the putative catalytic region of each of the three domains and is therefore devoid of the classical Trx or Grx catalytic activities.

\section{(c) The Author(s) 2019}

(c) (i) Open Access This article is licensed under a Creative Commons Attribution 4.0 International License, which permits use, sharing, adaptation, distribution and reproduction c. in any medium or format, as long as you give appropriate credit to the original author(s) and the source, provide a link to the Creative Commons license, and indicate if changes were made. The images or other third party material in this article are included in the article's Creative Commons license, unless indicated otherwise in a credit line to the material. If material is not included in the article's Creative Commons license and your intended use is not permitted by statutory regulation or exceeds the permitted use, you will need to obtain permission directly from the copyright holder. To view a copy of this license, visit http://creativecommons.org/licenses/by/4.0/. 
While the exact biological role of PICOT has not yet been resolved, its critical role in embryonic development has been validated in PICOT deficient mice that showed growth retardation and morphological defects during development, leading to death at $\sim 12.5$ days post coitum ${ }^{4,5}$.

PICOT is a ubiquitously expressed protein that resides predominantly in the cytoplasm ${ }^{6}$. However, it is also found in cell nuclei ${ }^{6}$, and its subcellular distribution at a given time depends on the cell type and the stage of activation/differentiation ${ }^{7}$.

PICOT regulates the c-Jun N-terminal kinase (JNK)/ AP-1 and NF- $\mathrm{KB}$ pathways in T cells, where it colocalizes with $\mathrm{PKC}^{1}$. Its expression is upregulated in cardiac muscles in response to hypertrophic stimuli where PICOT attenuates the pressure overload-induced cardiac hypertrophy and increases ventricular function and cardiomyocyte contractility ${ }^{5,8}$. Further studies demonstrated that PICOT plays a role in cellular iron metabolism and biogenesis of $\mathrm{Fe} / \mathrm{S}$ proteins and hemoglobin maturation $^{9,10}$. PICOT expression levels are augmented in mitogen-stimulated or antigen-stimulated $\mathrm{T}$ cells ${ }^{11}$, as well as in various tumor cell lines ${ }^{11}$. In addition, high expression levels of PICOT were observed in vivo in a variety of cancers, including Hodgkin's lymphoma ${ }^{11}$ and breast $^{7}$, colon, and lung carcinoma ${ }^{12}$.

Utilization of a full-length human PICOT cDNA in a yeast two-hybrid screen of a Jurkat $T$ cell cDNA library revealed that PICOT interacts with the embryonic ectoderm development (EED) protein ${ }^{13}$. PICOT interaction with EED was verified in various human cell lines and reconfirmed using GST pull-down assays, reciprocal coimmunoprecipitation and immunofluorescence imaging $^{13}$. Binding of PICOT to EED is mediated by each of its two C-terminal PICOT/Grx homology domains ${ }^{13}$.

EED is a member of the Polycomb-Group (PcG) proteins $^{14,15}$ that are critical for chromatin remodeling and epigenetic gene silencing ${ }^{16}$. EED serves as a core component of the polycomb repressive complex 2 (PRC2) which catalyzes histone $\mathrm{H} 3$ trimethylation on lysine 27 (H3K27me3), a mark of transcriptional repression of multiple genes ${ }^{17}$.

Based on the above information, we hypothesized that PICOT interaction with EED might have an impact on

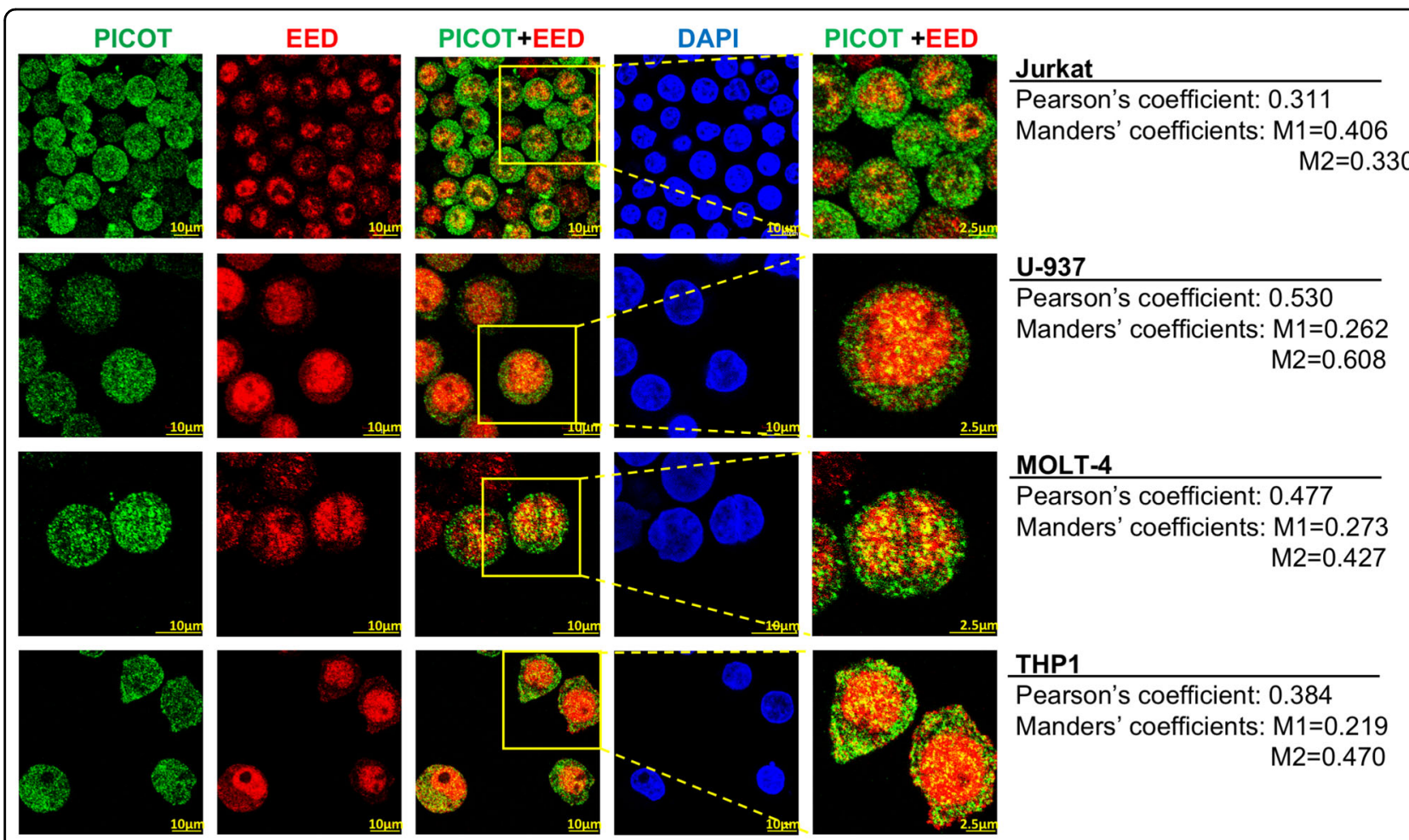

Fig. 1 Nuclear PICOT partially colocalizes with EED in leukemia cells. Jurkat, U-937, MOLT4 and THP1 cells (1.5 $\times 10^{6} /$ group) were seeded on poly-L-lysine-coated 8 well $\mu$-slide (ibidi Ltd.) and deposited by centrifugation at $1200 \mathrm{rpm}$ for $5 \mathrm{~min}$. The cells were fixed, permeabilized and incubated with a mixture of mouse anti-PICOT mAbs and rabbit anti-EED polyclonal Abs for $1 \mathrm{~h}$ at room temperature. The cells were then stained using Cy3-conjugated anti-mouse Abs and Cy5-conjugated anti-rabbit Abs, plus nuclei counterstain with DAPI (1 $\mu \mathrm{g} / \mathrm{ml}$ in PBS) for $1 \mathrm{~h}$ in the dark, at room temperature. The cells were then analyzed using a confocal microscope (Olympus Flouview 1000 laser scanning confocal microscope). PICOTEED colocalization is demonstrated in a color overlay panel, and a selected area marked by a red box that was magnified is shown in the right panel. Comparative quantification of PICOT-EED colocalization was performed using the ImageJ colocalization JACoP imageJ plugin ${ }^{18}$. Pearson's coefficient value and Manders' coefficient values ( $M 1=$ red overlap with green; $M 2=$ green overlap with red) are indicated on right for each panel 


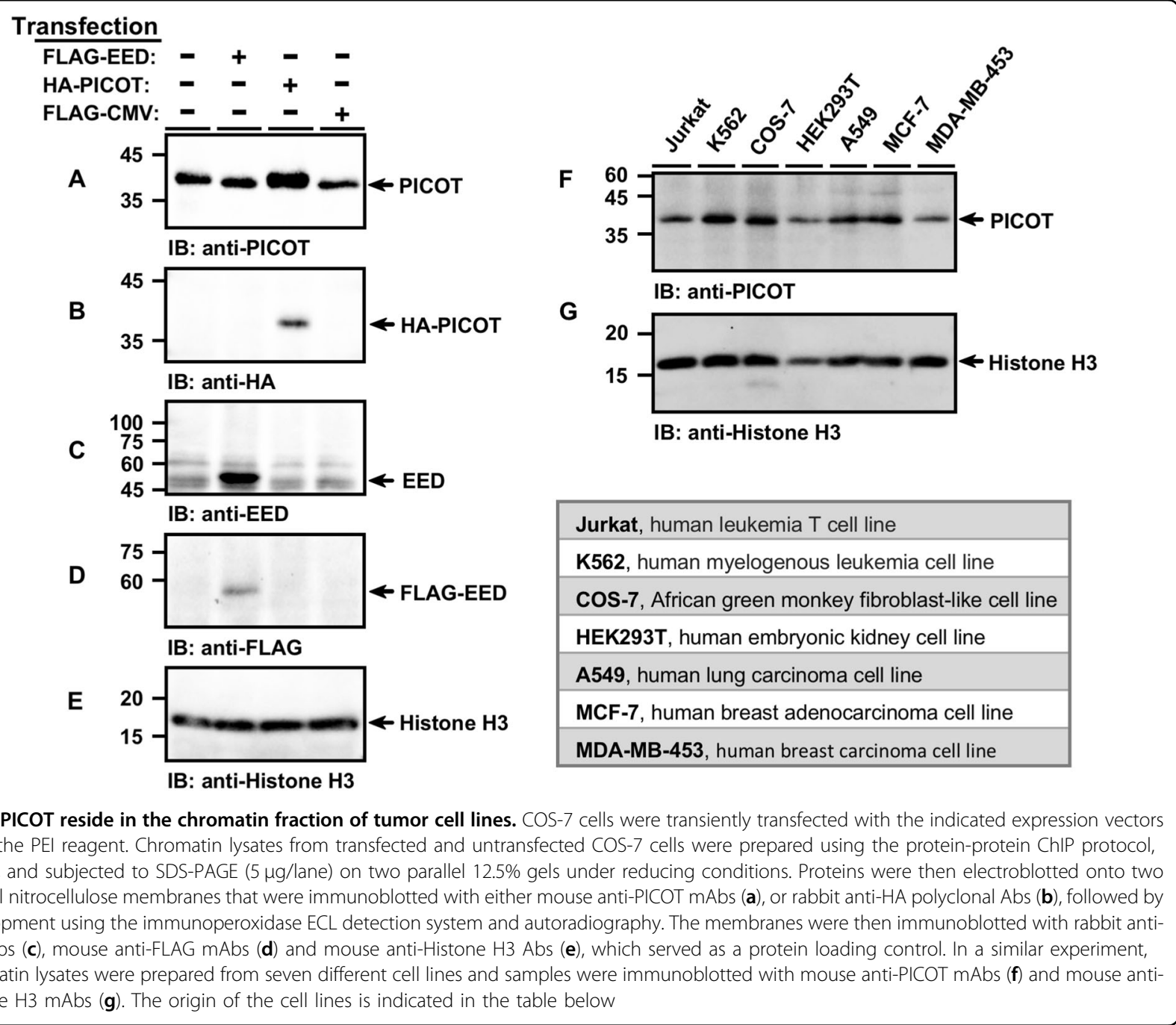

transcriptional processes of PRC2 target genes. Initial studies supported this hypothesis by demonstrating that PICOT knock-down in Jurkat $\mathrm{T}$ cells led to a reduced H3K27me3 at the PRC2 target gene, myelin transcription factor $1(M Y T 1)^{13}$.

In the present study, we further analyzed the potential involvement of PICOT in the regulation of PRC2mediated H3K27 trimethylation. We also analyzed the effect of PICOT on the transcription and translation of selected PRC2 target genes and tested the potential relevance of this regulation to cancer progression.

\section{Results}

Nuclear PICOT partially colocalizes with EED in leukemia cells

To analyze whether PICOT colocalizes with nuclear EED in leukemia cells, we performed immunofluorescence staining of PICOT and EED in four different human leukemia cell lines. Laser scanning confocal microscopy of the stained cells reconfirmed the predominant nuclear localization of EED and demonstrated that PICOT resides in both the cytoplasm and nucleus (Fig. 1). Interestingly, overlay images revealed partial colocalization of the two proteins predominantly in the nuclei of all cell lines (Fig. 1), suggesting that PICOT and EED might function together in the nucleus. The extent of PICOT-EED colocalization in each of the cell lines was calculated using the ImageJ colocalization JACoP imageJ plugin $^{18}$ and values are shown in Fig. 1.

Specificity of the anti-PICOT Abs was verified by the lack of immunofluorescence staining of PICOT-deficient Jurkat $\mathrm{T}$ cells (Jurkat.1A) (not shown), whereas the specificity of the anti-EED Abs was demonstrated on EEDdeficient Jurkat $\mathrm{T}$ cells obtained by transfection of EEDspecific small interfering (si) $\mathrm{RNA}^{19}$.

PICOT reside in the chromatin fraction of tumor cell lines

As the nuclear PICOT colocalizes with EED, a core component of PRC2, which associates with chromatin and 
maintains its repressive state ${ }^{13}$, we tested whether PICOT can also associate with chromatin.

COS-7 cells were transiently transfected with $H A$ PICOT, FLAG-EED, or a control FLAG-CMV expression vector followed by chromatin isolation, as indicated in the standard chromatin immunoprecipitation (ChIP) assay. Western blot analysis revealed that the chromatin fraction included both endogenous and heterologous PICOT proteins (Fig. 2a, b), as well as endogenous and heterologous EED proteins (Fig. 2c, d).

To further analyze whether the presence of PICOT in chromatin lysates is a general phenomenon, we isolated chromatin from seven different cell lines followed by SDSPAGE fractionation. Western blot analysis revealed that PICOT resides in the chromatin fraction of all tested cell lines (Fig. 2f), suggesting a functional role for nuclear PICOT in chromatin regulation.

\section{PICOT associate with chromatin residing EED}

To test whether PICOT interacts with chromatinassociated EED, we performed specific coimmunoprecipitation on chromatin fraction of Jurkat $\mathrm{T}$ cell lysates using PICOT-specific Abs. We found that EED coimmunoprecipitated with PICOT, while normal IgG that served as a control did not immunoprecipitate EED (Fig. 3a). In contrast, anti-PICOT Abs did not coimmunoprecipitate other PRC2 core components, such as EZH2 (Fig. 3b) or SUZ12 (Fig. 3c).

As a technical control, PICOT immunoprecipitates that were immunoblotted with anti-PICOT Abs showed efficient recovery of PICOT proteins from chromatin lysates (Fig. 3d). Furthermore, these results were repeated using COS-7 cells, where EED (Fig. 3f), but not EZH2 (Fig. 3g) coimmunoprecipitated with PICOT.

To further substantiate these findings, we performed a reciprocal coimmunoprecipitation on Jurkat $\mathrm{T}$ cell chromatin lysates and found that anti-EED-Abs coimmunoprecipitated both PICOT (Fig. 1sA) and EZH2 (Fig. 1sB). The reciprocal coimmunoprecipitation studies (Fig. 1s) suggest that the chromatin-associated EED can interact with both PICOT and EZH2. However, the PICOTassociated EED does not bind EZH2 (Fig. 3), suggesting that PICOT binds chromatin-associated EED which is free of the other PRC2 core proteins.

\section{Knockdown of PICOT results in reduced association of H3K27me3, EED and EZH2 at the CCND2 gene promoter}

Since PICOT association with EED occurs at the chromatin level, we tested whether this interaction can alter the PRC2-mediated H3K27me3 levels at PRC2 target genes. Using ChIP-qPCR, we compared the levels of trimethyl H3K27 at selected target genes in chromatin lysates of control vs. PICOT-deficient Jurkat $\mathrm{T}$ cells.
We initially tested the expression levels of PICOT in the two cell lines and found that PICOT-deficient Jurkat $\mathrm{T}$ cells (Jurkat.1A) express $\sim 5$-fold lower levels of PICOT compared to the control Jurkat cells (Fig. 4a).

Next, we performed ChIP-qPCR using H3K27me3 and IgG isotype (as a control) and analyzed the H3K27me3 enrichment at selected genomic regions that include three PRC2 target genes: Cyclin-D2 (CCND2), Homeobox A2 (HOXA2) and Activating transcription factor 3 (ATF3), as well as Cell division cycle 6 (CDC6), a non-PRC2 target gene that serves as a negative control. We found a $\sim 3.5$ fold significant H3K27me3 enrichment $(p<0.0001)$ on the promoter of CCND2 in Jurkat T cells when compared to the PICOT-deficient cells (Fig. 4d). In contrast, no H3K27me3 enrichment was observed at HOXA2 and $A T F 3$, or at the PRC2 non-target gene, CDC6 (Fig. 4d).

The fact that DNA enrichment was not general, and observed at selected genes (CCND2) was not surprising, since desilence of selected PRC2 target genes has already been reported following knockdown of genes of various PRC2-associated proteins ${ }^{20}$.

Furthermore, when the ChIP-qPCR was optimized for the PRC2 components, EZH2 and EED, the percentage input value for the PRC2 target genes was significantly higher when using either anti-EED (Fig. 2sB) or antiEZH2 Abs (Fig. 2sC), compared to isotype control.

The percentage input values for the PRC2 non-target genes, $\alpha$-satellite, were similar when either anti-EED (Fig. 2sB) or anti-EZH2 Abs (Fig. 2sC) were compared to isotype control.

Since the trimethylation of H3K27 is catalyzed by $\mathrm{EZH} 2^{20,21}$ and further propagated by the help of $\mathrm{EED}^{22,23}$, we further tested whether PICOT knockdown might effect EZH2 and EED presence at PRC2 target genes.

Using ChIP-qPCR with anti-EZH2 Abs, we revealed a $\sim 2.5$-fold lower enrichment of EZH2 at the CCND2 promoter in PICOT deficient cells (Fig. 4e).

Moreover, ChIP-qPCR with anti-EED Abs showed a 3fold lower enrichment of EED at the CCND2 promoter in PICOT deficient cells, compared to control Jurkat cells (Fig. 4f). In both EZH2 and EED ChIP analyses, no enrichment was observed at HOXA2 and $\alpha$-satellite in PICOT deficient cells (Fig. 4e, f).

Thus, PICOT associates with EED and promotes the trimethylation of $\mathrm{H} 3 \mathrm{~K} 27$ on the CCND2 promoter, and might therefore have an effect on the expression of CCND2.

To test whether PICOT might be directly associated with the CCND2 promoter region we performed ChIPqPCR using either anti-PICOT mAbs, to immunoprecipitate the endogenous PICOT protein from Jurkat cell chromatin lysates, or ChIP-grade anti-HA Abs to immunoprecipitatie a transiently expressed HA-PICOT from COS-7 cell chromatin lysates. Results demonstrated that 


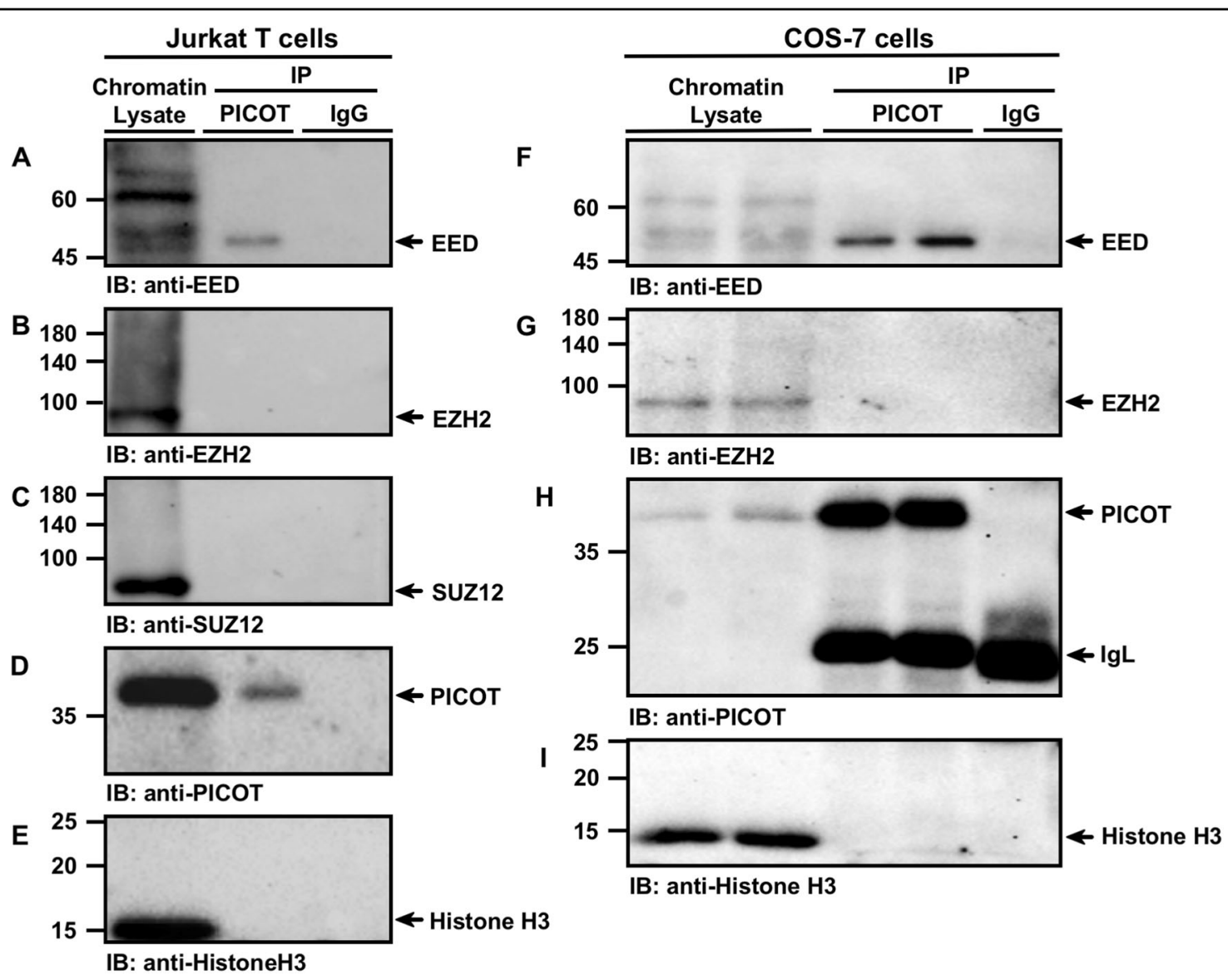

Fig. 3 PICOT-EED interaction co-exists in the chromatin compartment. Jurkat and COS-7 cells $\left(20 \times 10^{6} /\right.$ group) were fixed in $1 \%$ formaldehyde for $10 \mathrm{~min}$ at room temperature and chromatin lysates were prepared using the protein-protein ChIP protocol. The chromatin samples were precleared on protein $\mathrm{G}$-sepharose beads, incubated overnight at $4{ }^{\circ} \mathrm{C}$ with protein $\mathrm{G}$ sepharose bead-immobilized mouse anti-PICOT mAbs or mouse normal $\operatorname{lgG}\left(10 \times 10^{6}\right.$ cell equivalent per group). Proteins were then eluted from the beads by resuspension of the samples in $\beta$-mercaptoethanol-containing sample buffer $(180 \mu \mathrm{l})$ and boiling for $30 \mathrm{~min}$. Following centrifugation, supernatants $\left(1.5 \times 10^{6}\right.$ cell equivalent in $\left.20 \mu \mathrm{l}\right)$ were subjected to SDS-PAGE on $10 \%$ gel under reducing conditions. Chromatin lysates ( $5 \mathrm{\mu g} / \mathrm{lane}$ ) were boiled and electrophoresed in parallel. Proteins were then electroblotted onto nitrocellulose membranes that were sequentially immunoblotted with rabbit anti-EED polyclonal Abs (a, $\mathbf{f}$ ) and rabbit anti-EZH2 mAbs $(\mathbf{b}, \mathbf{g})$. A parallel membrane was sequentially immunoblotted with rabbit anti-SUZ12 mAbs (c), mouse anti-PICOT mAbs $(\mathbf{d}, \mathbf{h})$ and mouse anti-histone $\mathrm{H} 3 \mathrm{mAbs}(\mathbf{e}, \mathbf{i})$. Protein bands were then visualized using the immunoperoxidase ECL detection system and autoradiography. Immunoprecipitation using IgG mAbs served as a negative control. The position of specific protein bands is indicated by arrows. Results are representative of three independent experiments. IP, immunoprecipitation; IB, immunoblot

the percentage input value for CCND2 using anti-PICOT mAbs (Fig. 3sA) or anti-HA Abs (Fig. 3sB) was not significantly different from that of the 'beads only' control group, suggesting that PICOT is not direclty associated with the CCND2 promoter.

Knock down of PICOT expression in Jurkat $\mathrm{T}$ cells increases cyclin D2 mRNA and protein expression levels

Since PRC2-mediated trimethyation of H3K27 at specific gene loci can negatively regulate gene transcripiton, we tested whether the lower levels of EED, EZH2, and $\mathrm{H} 3 \mathrm{~K} 27 \mathrm{me} 3$ at the CCND2 promoter in PICOT-deficient cells would also be reflected at the gene transcription and translation levels.
Using qRT-PCR, we tested the expression levels of CCND2 in control and PICOT-deficient cells. The two PICOT-deficient sublines, Jurkat.1A and Jurkat.2G exhibited significantly higher expression levels of CCND2 mRNA compared to the levels observed in control Jurkat $\mathrm{T}$ cells, or in Jurkat.5A subline, which expresses the control scrambled sc-PICOT DNA (Fig. 5a).

Increased CCND2 mRNA levels in PICOT-deficient cells were not due to altered RNA-polymerase II (Pol II) levels at the CCND2 locus, since RNA-pol II ChIP data revealled similar levels of RNA-pol II at the CCND2 upstream promoter region in both wild type and PICOTdeficient Jurkat $\mathrm{T}$ cells (not shown). 


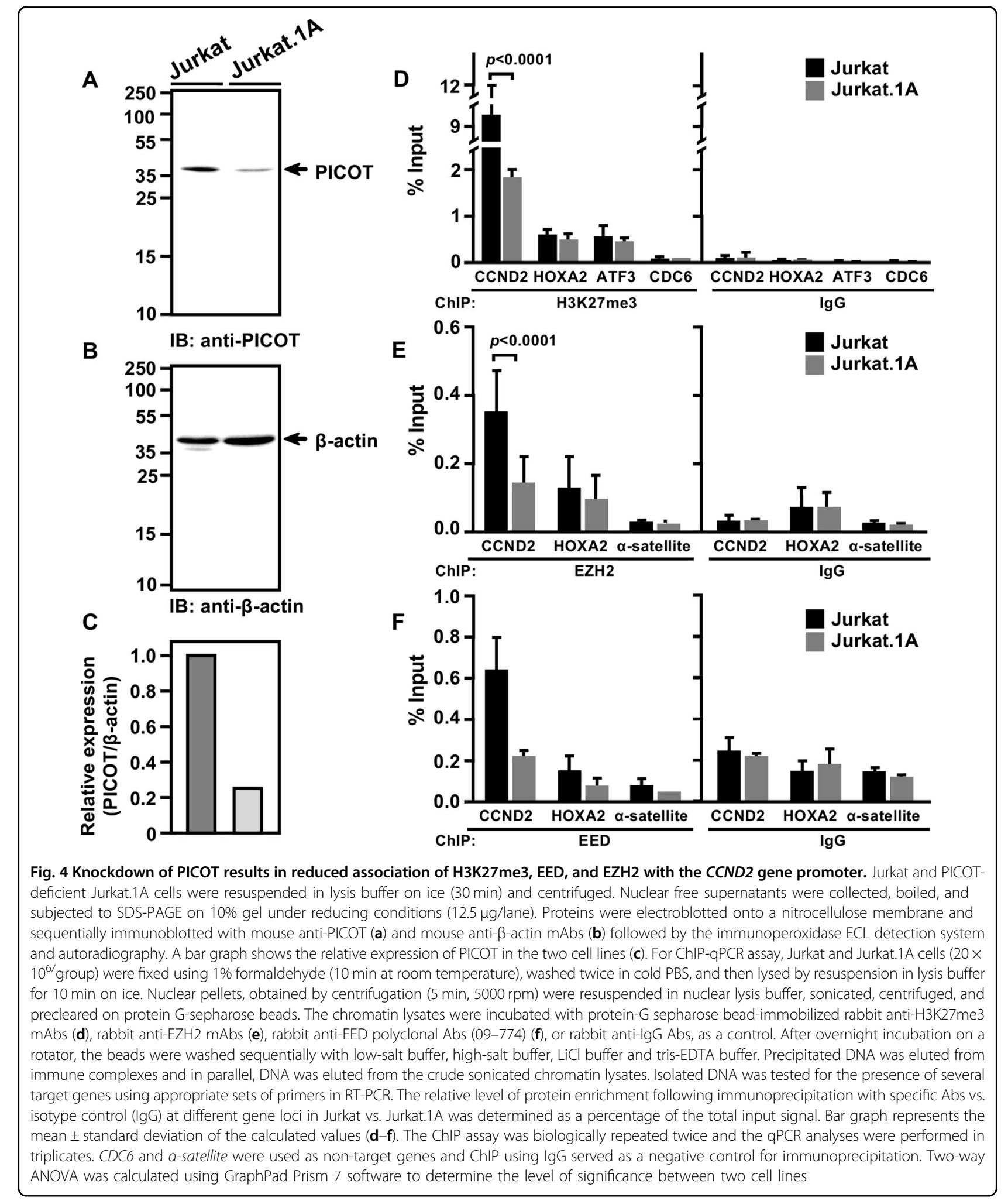

Furthermore, determination of the levels of the CCND2-encoding protein, cyclin D2, in PICOT-deficient cells revealed high levels of protein expression in both
PICOT-deficient cells, Jurkat.1A and Jurkat.2G, whereas cyclin D2 protein expression levels in Jurkat and Jurkat.5A were almost undetectable (Fig. 5b). 


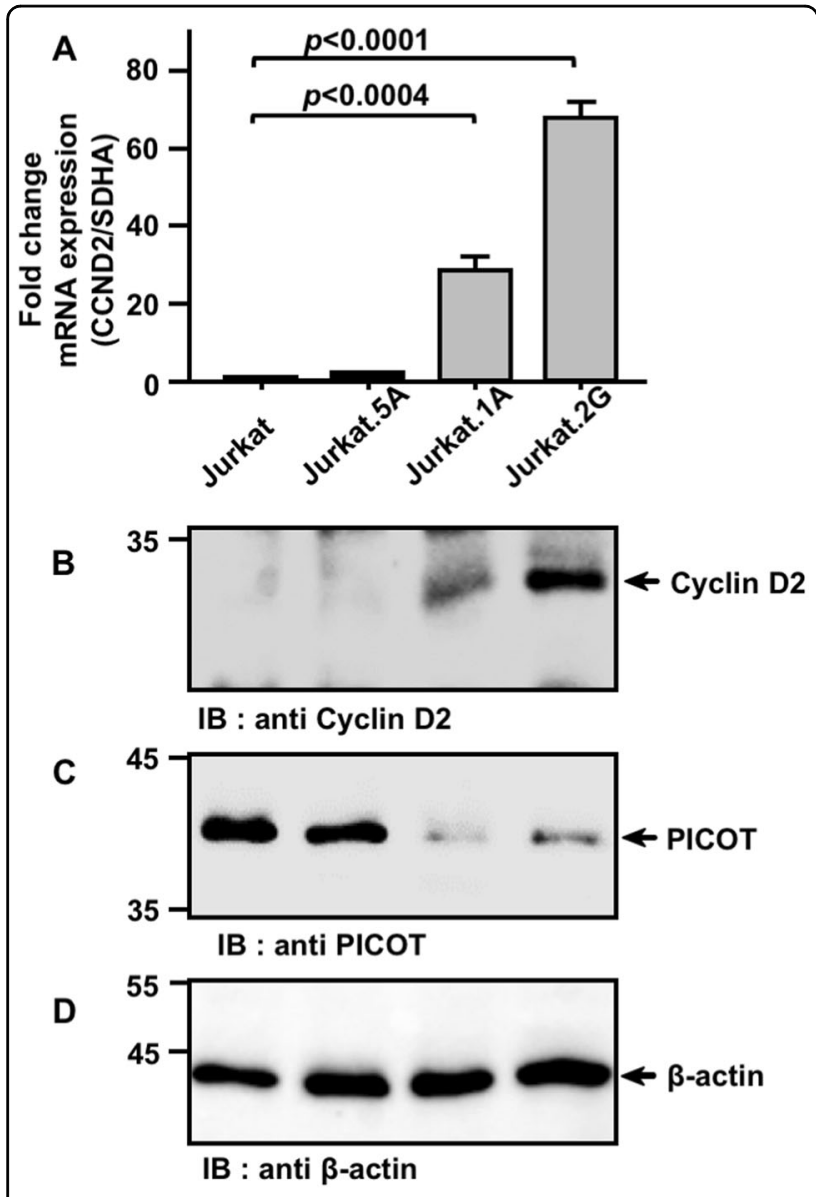

Fig. 5 PICOT expression in Jurkat T cells negatively correlates with CCND2 mRNA and protein expression. a. RNA was extracted from Jurkat cells, PICOT-deficient sh-PICOT-expressing Jurkat sublines (1A and $2 \mathrm{G})$, and a control, sc-PICOT expressing Jurkat subline (5A). Total RNA from each sample (1 $\mu \mathrm{g} / \mathrm{sample})$ was isolated and served as a template for complementary DNA (CDNA) preparation using the Bio$\mathrm{RT}^{\mathrm{TM}}$ first strand CDNA synthesis kit (Bio-Lab) plus random hexamer primers. The CDNA generated during reverse transcription then served as template in the subsequent qRT-PCR that was carried out in the presence of CCND2 or SDHA gene-specific primers and the doublestrand DNA intercalating fluorescent dye, SYBR ${ }^{\circledR}$ green. The relative fold change in CCND2 mRNA was determined by the ${ }^{\triangle \triangle} \mathrm{Ct}$ method and calculated relative to the SDHA mRNA. The results are presented as a bar graph where mean \pm standard error are indicated. Real-time PCR analyses were biologically repeated thrice with triplicate wells in each experiment. One-way ANOVA test was calculated using GraphPad Prism 7 software to determine the level of significance. $\mathbf{b}-\mathbf{d}$. For Western blot analysis, Jurkat and Jurkat-derived subclones $\left(5 \times 10^{6}\right.$ cells/group) were resuspended in lysis buffer on ice (30 min), centrifuged and nuclear free supernatants were collected. Cell lysates ( $2.5 \mu \mathrm{g} /$ lane) were boiled and subjected to SDS-PAGE on $10 \%$ gels under reducing conditions. Proteins were then electroblotted onto a nitrocellulose membrane, immunoblotted with rabbit anti-Cyclin D2 mAbs (b) followed by immunoperoxidase ECL detection system and autoradiography. Parallel membranes were immunoblotted with mouse anti-PICOT mAbs (c) and mouse anti- $\beta$-actin mAbs (d). The position of specific protein bands is indicated by arrows. Results are representative of three independent experiments
Western blot studies reconfirmed the lower levels of the PICOT protein in Jurkat.1A and Jurkat.2G cells (Fig. 5c), while the equal loading of lysate/lane was verified using anti- $\beta$-actin mAbs (Fig. 5 d).

\section{PICOT expression levels negatively correlates with CCND2 expression levels in human cancers}

The current findings indicate that PICOT deficiency in Jurkat $\mathrm{T}$ cells correlates with hypomethylation of H3K27 at the CCND2 promoter, which results in upregulation of CCND2 gene transcription and translation, suggesting that PICOT might have a negative regulatory effect on the synthesis of cyclin D2. To analyze whether such a correlation might exist in human cancers, we downloaded data on PICOT and CCND2 mRNA expression levels in 32 types of human cancers available at the Cancer Genome Atlas (TCGA) dataset. Using the Pearson's correlation coefficient, we examined the extent of correlation between PICOT and CCND2 in each cancer, after clipping the top and bottom decile of each measurement to avoid outlier effects (Fig. 6). The general tendency of negative correlation between the expression levels of PICOT and CCND2 mRNA levels can be observed (Table Is).

However, a negative and significant correlation between PICOT and CCND2 mRNA levels was only observed for 8 out of the 32 tumor types considered. Of these, the strongest correlation was observed in prostate adenocarcinoma (PRAD), lung adenocarcinoma (LUAD) and pancreatic adenocarcinoma (PAAD), with lower, but significant values in glioblastoma multiforme (GBM), breast invasive carcinoma (BRCA), gastric adenocarcinoma (STAD), esophageal carcinoma (ESCA) and ovarian cancer (OV). Subsequent analysis was conducted on 5 cancer types.

The correlation between PICOT and CCND2 could be specific to cancer, reflecting abnormal regulation of the transformed cells, or could be universal and reflect basic cellular control mechanisms. To differentiate between these two hypotheses, we attempted to test the PICOTCCND2 correlation in normal tissues which are adjacent to the tumor (normal adjacent tissue; NAT), and compare them with the results obtained in the tumors. However, information on PICOT and CCND2 mRNA expression levels in NAT samples was much less abundant and enabled statistical analysis only in BRCA $(n=96)$ and LUAD $(n=54)$. We found that, in contrast to the results observed in BRCA, where PICOT and CCND2 mRNA levels exhibited a weak but significant negative correlation (Cor $=-0.0971, \quad p=0.001), \quad$ an opposite and nonsignificant correlation was observed in BRCA NAT (Cor $=$ $+0.03684, p=0.721)$. The negative correlation between PICOT and CCND2 mRNA expression levels that was observed in LUAD repeated also in LUAD NAT (Fig. 4s).

To analyze whether an opposite correlation exists between PICOT and CCND2 mRNA levels in LUAD vs. 


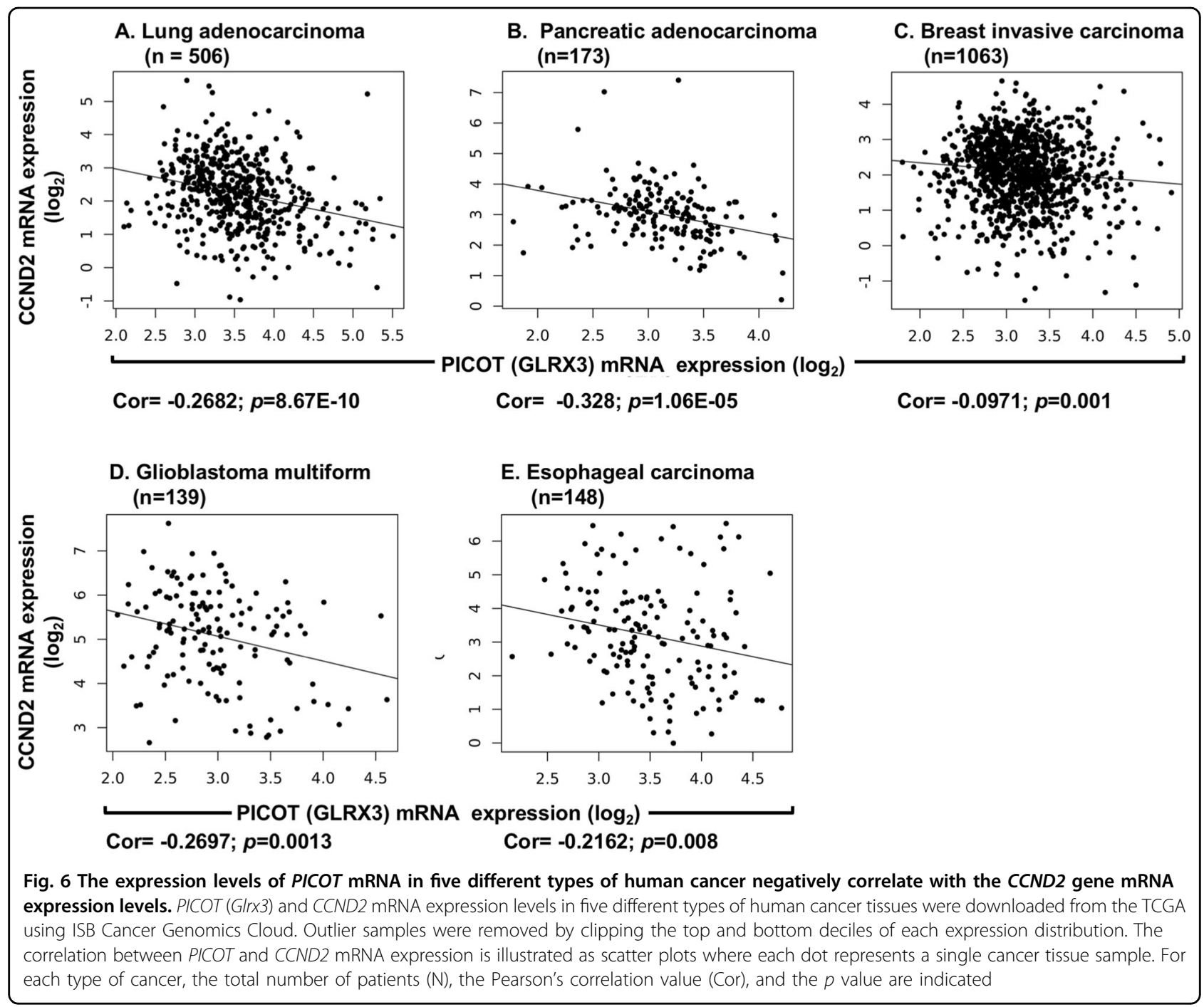

LUAD NAT, and test the generality of this phenomenon, we used again data from the TCGA, and compared PICOT and CCND2 mRNA expression levels in LUAD vs. LUAD NAT. The results (Fig. 5s) demonstrated a highly significant increase in PICOT mRNA expression in LUAD compared to LUAD NAT, and a highly significant decrease in CCND2 mRNA expression in LUAD compared to LUAD NAT suggesting that increased PICOT and decreased CCND2 mRNA levels can serve as a tumor marker in LUAD.

An independent analysis performed on T-ALL data from GSE62156, indicated a correlation of $-0.3(p=0.016, N=$ 64; $t$-test on Pearson's product moment correlation coefficient) between PICOT and CCND2 expression.

A relatively high expression of PICOT and low expression of CCND2 correlates with poor patient survival in five different types of human cancers

Previous studies suggested that high expression levels of PICOT correlate with growth promotion of normal ${ }^{11}$ and cancer $^{11,12,24}$ cells, whereas increased expression of Cyclin D2 negatively affects the growth of prostate cancer cells ${ }^{25}$. Since our data demonstrated a negative correlation between PICOT and cyclin D2 mRNA expression levels in selected types of human cancers, we further analyzed the impact of such a correlation on the overall patients' survival. Using publicly available TCGA datasets, the gene expression data and the patients' clinical outcome were obtained for five types of cancer diseases, namely, lung adenocarcinoma (LUAD), breast invasive carcinoma (BRCA), pancreatic adenocarcinoma (PAAD), glioblastoma multiforme (GBM), and esophageal carcinoma (ESCA). The patients were divided into two groups based on the gene expression levels, where the top and bottom tertiles, in terms of gene expression, were considered as "high expressers" and "low expressers", respectively.

The Kaplan-Meier survival plots for gene expression vs. survival probability and the log-rank $p$ value, as well as the hazard ratio in each type of tumors, were compared 


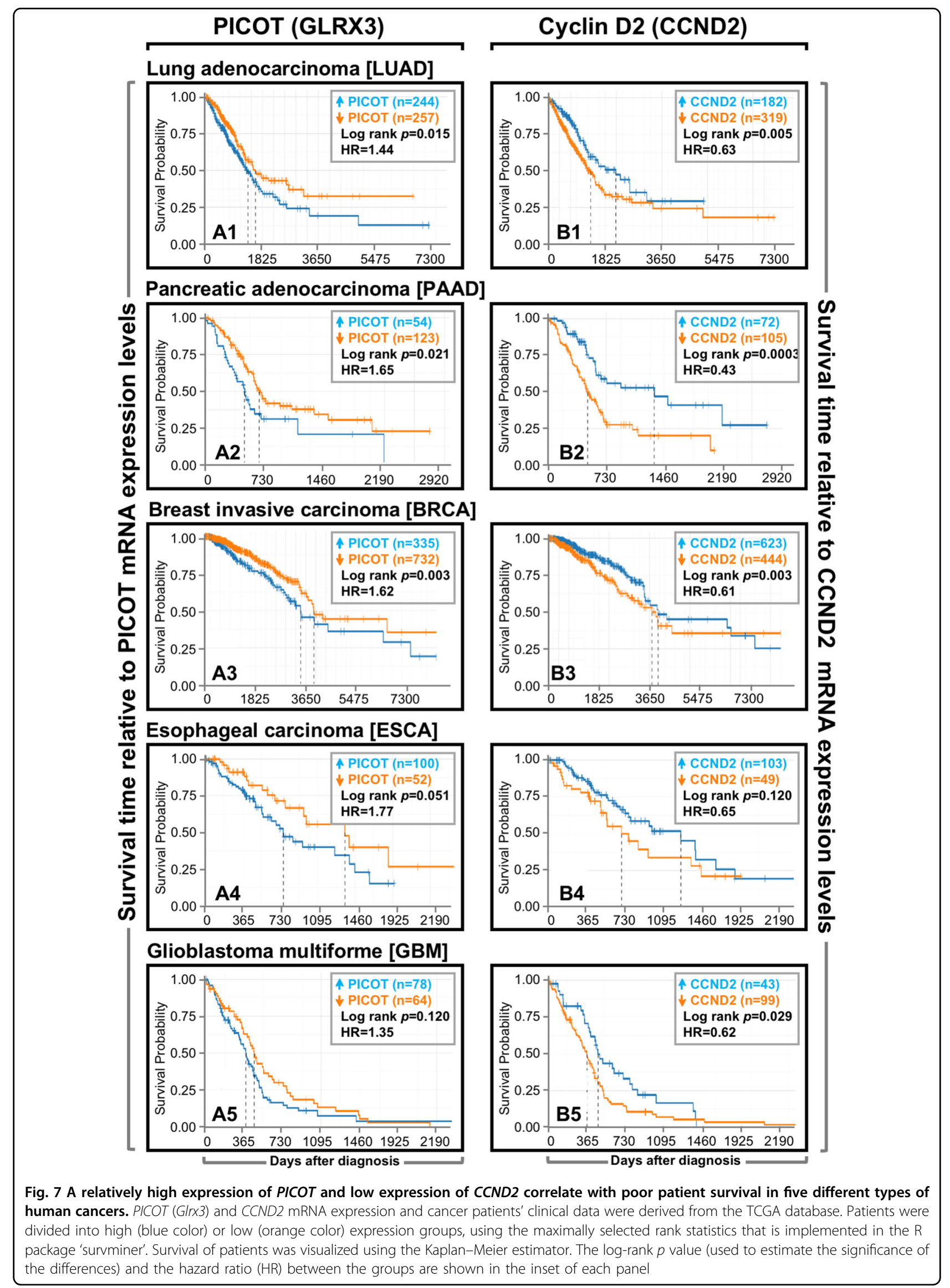


(Fig. 7). The results demonstrate a significant log-rank $p$ value for PICOT in LUAD, PAAD, BRCA, and ESCA (Fig. 7A1-4). Similar tendency was observed for the log rank $p$ value in GBM (Fig. 7A5), although the correlation with PICOT expression and survival probability $(0.11)$ was statistically insignificant. Furthermore, a high hazard ratio $(>1)$ in relation to PICOT expression was observed in all tumor patients. When the $\log$ rank $p$ value was obtained for cyclin $D 2$ low vs. high expressers, it was statistically significant in LUAD, PAAD, BRCA, and GBM (Fig. 7B1-4), but not in ESCA (Fig. 7B5), and a low hazard ratio $(<1)$ was observed in all tumor types. Absence of clinical data for the T-ALL patients did not allow to analyze the linkage between PICOT expression and patient survival in this cohort.

Thus, our results suggest that overexpression of PICOT and downregulation of $C C N D 2$ correlate with poor overall patient survival in the majority of analyzed tumors.

\section{Discussion}

The observation that PICOT deficiency results in embryonic lethality ${ }^{4,5}$ implicates PICOT in cellular functions that are critical for embryogenesis. In addition, PICOT knockdown led to a slower growth rate of Jurkat $\mathrm{T}$ cells (Fig. 6s) and impaired both cell cycle progression and multiplication of mouse fibroblasts and HeLa cells ${ }^{4}$. Furthermore, higher expression levels of PICOT were observed in T lymphocytes ${ }^{11}$ and cardiac muscle cells ${ }^{8,26}$ following their stimulation. Nevertheless, the molecular mechanisms by which PICOT affects cell growth and modulates cell cycle progression are not yet clear.

In a previous study, we demonstrated that PICOT can associate with EED and that both proteins can partially colocalize at the cell's nuclei ${ }^{13}$. Furthermore, PICOT binding to EED had a positive effect on H3K27 methylation at the MYT1 PRC2 target gene ${ }^{13}$. Based on these findings, we suggested that PICOT binding to EED might have an impact on the assembly or coordination of the PRC2 protein subunits, modulate the EED-dependent EZH2-mediated histone $\mathrm{H} 3$ lysine 27 trimethylation, and contribute to the overall epigenetic regulation of chromatin silencing and remodeling.

In the present work, we found a first link between PICOT function and cell cycle regulation by demonstrating that PICOT binding to EED modulates the transcriptional regulation of the PRC2 target gene, $C C N D 2$, a cell cycle-regulating gene which encodes the cyclin D2 protein.

We demonstrated that PICOT associates with the chromatin-residing EED and that PICOT knock-down leads to a reduced $\mathrm{H} 3 \mathrm{~K} 27 \mathrm{me} 3$ mark and a decrease in EED and EZH2 at the CCND2 gene promoter. These events were accompanied by a marked rise in CCND2 mRNA and protein expression. We hypothesize that increased levels of cyclin D2 might alter the activity of its target genes, such as $C D K 4$ and $C D K 6^{27}$, modulate the cell cycle progression ${ }^{28}$, and promote nuclear mechanisms leading to early embryonic cell cycle arrest ${ }^{29}$.

PICOT knockdown did not alter the global levels of H3K27me3 (not shown), as was demonstrated for other PRC2 auxiliary proteins, such as Jumonji and AT-rich interaction domain containing 2 (JARID2) ${ }^{30}$. A difference in the effect of PICOT knockdown on the two PRC2 target genes, CCND2 and HOXA2, is not surprising, since knockdown of other PRC2 associated proteins, such as the Polycomb-like proteins (PCL), were also found to desilence only selected PRC2 target genes ${ }^{20}$.

EED is a core component of $\mathrm{PRC} 2^{17}$, and its major biological activity is in connection with the PRC2 complex-mediated histone methyltransferase activity, which promotes epigenetic gene silencing. The critical role of EED in embryonic development was demonstrated in knock out mice that died on day $\sim 12.5$ post implantation $^{31-33}$.

Along with EED, the PRC2 complex possesses three additional core components, namely zeste homolog 1 or 2 (EZH1/2), suppressor of zeste12 (SUZ12) and retinoblastoma protein-associated protein 46/48 (RbAp46/48). EZH1 and EZH2 are homologous proteins that serve as the PRC2 catalytic subunits that mediate the methyltransferase activity. While both proteins promote transcriptional silencing, they maintain repressive chromatin through different mechanisms ${ }^{34,35}$. Jurkat $\mathrm{T}$ cells express only a very small amount of EZH1 that resides in the cytoplasm, where it can interact with ZAP70 ${ }^{36}$. In contrast, EZH2 which resides in the nucleus, is the predominant isoform in Jurkat $\mathrm{T}$ cells $\mathrm{s}^{37,38}$ and its expression is associated with cell proliferation ${ }^{34}$.

EZH2 requires both EED and SUZ12 in order to maintain the PRC2 complex integrity and histone methyltransferase activity ${ }^{23,39}$. EED binding to EZH2 is mediated by the EED-WD40 domain ${ }^{37}$, and upon interaction with H3K27me3, EED recruits PRC2 complexes to established H3K27me3 and enforces the maintenance of the repressive epigenetic mark $^{17}$.

Mammalian EED includes four distinct isoforms produced by alternative utilization of four distinct, in-frame translation start sites in the Eed mRNA ${ }^{21,40}$. Usage of the EED isoforms is regulated developmentally, suggesting that distinct EED isoform-containing PRC2 complexes may differ in their histone methyltransferase activity and/ or target selectivity ${ }^{21}$. Differential association of PICOT with selected EED isoforms may thus have distinct effects on the activity and specificity of the PRC2-mediated trimethylation of H3K27.

The assembly, composition and biological activity of PRC2 complexes appear to be very complicated due to the potential interaction of the four core components of 
PRC2 with several additional auxiliary proteins ${ }^{41,42}$. A recent study indicated that PRC2 can associate with two alternative sets of proteins, namely PALI1/PCL1-3 or AEBP2/JARID2, which define two mutually exclusive antagonistic PRC2 subtypes, termed PRC2.1 and PRC2.2, respectively ${ }^{43}$. These two complexes exhibit divergent H3K27-methylation activities and regulate repression of different sets of polycomb target genes ${ }^{43}$. It is possible, therefore, that differential interaction of PICOT with PRC2.1-associated or PRC2.2-associated EED may have different impacts on cellular epigenetic mechanisms that alter distinct cell-specific gene expression signatures. While the nature of the EED isoforms that associate with each of the two PRC2 subtypes has not yet been determined, the existence of four distinct EED isoforms may add to the complexity and heterogeneity of this epigenetic mechanism.

Downregulation of CCND2 mRNA was reported as a striking feature of several human tumors, including the lung $^{44}$, breast ${ }^{45,46}$ and pancreas ${ }^{47}$ tumor tissues. This effect reflected hypermethylation at the CCND2 gene promoter region which is rarely observed in NAT samples $^{44-47}$. In agreement with these findings, we observed an increased hypermethylation at the CCND2 promoter and reduced cyclin D2 expression in wild type Jurkat cells, whereas PICOT-deficient Jurkat cells exhibited reduced methylation and increased expression of cyclin D2.

Targeting of CCND2 in non-small-cell lung carcinoma cells with the microRNA miR-146a-5p, inhibited cell cycle progression and cell proliferation ${ }^{48}$, and other CCND2 targeting miRs inhibited the proliferation of additional types of cancer cells ${ }^{49-51}$. It appears, therefore, that downregulation of CCND2 is required for cell cycle progression and replication of certain cell types, and that overexpression of PICOT can lead to excessive downregulation of CCND2 expression, which might promote the cell growth in several types of human cancers.

The increased expression of PICOT protein in a variety of cancers, including Hodgkin's lymphoma ${ }^{11}$ and breast ${ }^{7}$, colon $^{12}$, and lung carcinoma ${ }^{12}$, encouraged us to test whether the negative correlation between PICOT and CCND2 expression, which was observed in Jurkat $\mathrm{T}$ cells, also exists in human cancers. Information obtained from the Cancer Genome Atlas (TCGA) database verified the negative correlation between PICOT and CCND2 in eight different human cancer types.

More important, high expression of PICOT and low expression of CCND2 correlated with poor patient survival in five different types of human cancers, namely lung and pancreatic adenocarcinoma, glioblastoma, and breast and esophageal cancer. Similar findings were observed in an independent validating dataset, using the Kaplan-Meir plotter, a web tool that reports difference in survival in an independent compendium of expression profiles from cancer patients (not shown). Interestingly, the existence of outliers in different cancer types (Fig. 6) suggests that additional mechanisms, besides PICOT-induced altered trimethylation of H3K27 at the CCND2 gene promoter, are likely to affect the cell cycle and growth rate of these cells.

The above information may imply that high expression of PICOT and low expression of CCND2 are advantageous to the growth of transformed cells in selected tissues, while other or additional factors affect the growth of transformed cells in other tissues.

\section{Materials and methods \\ Reagents and antibodies}

Mouse monoclonal antibodies (mAbs) specific for PICOT were from Santa Cruz Biotechnology, Inc. (Santa Cruz, CA). Mouse anti- $\beta$-actin mAbs and normal mouse IgG were from Merck Millipore (Darmstadt, Germany). Mouse anti-Histone $\mathrm{H} 3 \mathrm{mAbs}$ and anti-Flag mAbs and rabbit anti-hemagglutinin (HA) and anti-RNA polymerase II (Pol II; Ab26721) polyclonal Abs were from Abcam Biotechnology Company (Cambridge, UK). Mouse antiFlag mAbs (clone M2) were from Sigma-Aldrich, Israel. Rabbit anti-EED polyclonal Abs (Ab4469), which were used for Western blot analysis, and rabbit anti-EED Abs (09-774), which were used for ChIP-qPCR assay (09-774) were from Abcam and Merck Millipore, respectively. Rabbit mAbs anti-EZH2, anti-SUZ12, anti-trimethyl histone H3 (Lys 27, H3K27me3), anti-cyclin D2 and normal rabbit IgG were from Cell Signaling Technology Inc. (Danvers, MA). Horseradish peroxidase (HRP)-conjugated goat anti-mouse and anti-rabbit immunoglobulin Abs were from Abcam Biotechnology. Protein GSepharose ${ }^{\circledast}$, Fast Flow (P3296) was from Sigma-Aldrich, Israel. Cyanine 3 (Cy3)-conjugated goat anti-mouse IgG and Cyanine 5 (Cy5)-conjugated goat anti-rabbit IgG Abs were from Jackson ImmunoResearch Laboratories, Inc. (West Grove, PA) and from Kirkegaard and Perry Laboratories (KPL), respectively. DAPI was from Biotium, Inc. (Hayward, CA). The full-length PICOT cDNA with a human influenza hemagglutinin $(H A)$ tag in the pEF mammalian expression vector (HA-PICOT) has been previously described ${ }^{1}$. The $E E D_{2-441}$ cDNA introduced into the $p F L A G-C M V 2$ eukaryotic expression vector (FLAG-EED) and a mock plasmid (FLAG-CMV) were gifts of Dr. Dieter Adam (University of Kiel, Germany) ${ }^{19}$.

\section{Preparation of PICOT-deficient Jurkat T cell sublines}

PICOT shRNA plasmids were designed and synthesized by SuperArray Bioscience (Frederick, MD) and introduced into an expression vector (pGeneClip) that directs the synthesis of small hairpin RNAs (shRNAs) in mammalian cells under the control of the polymerase III promoter, and contains the neomycin resistance gene for 
selection of stably transfected cells. We used a set of four sense strands of 21 nucleotide sequences of human PICOT (CCAACATACCCTCAGCTGTAT; CTACCCAGCGCTAATGAACAT; GTGGAAATTCTTCACAAACAT; ACTCCCTCAAGTTTC ATTTGT), plus a control scrambled sequence. The sense strand was followed by a 10-nucleotide spacer (CTTCCTGTCA) and a reverse complement of the same 21 nucleotide sequence. After cloning these short sequences downstream of the U1 promoter of the plasmid, the resulting pGeneClipPICOT-shRNA plasmids were transfected into Jurkat E.6 cells by electroporation using a BioRad Gene Pulser. G418 resistant stable transfectants were isolated and cloned and their PICOT expression levels were determined by Western blot and qRT-PCR.

\section{Cell lines and culture conditions}

The Jurkat human T cell line (clone E6.1; ATCC ${ }^{\circ}$ TIB$152^{\mathrm{TM}}$ ), PICOT-deficient Jurkat-derived $\mathrm{T}$ cell lines (Jurkat.1A, Jurkat.2G), a control Jurkat cell line expressing a scramble PICOT plasmid (Jurkat.5A), THP1 human acute monocytic cell line (ATCC ${ }^{\circ}$ TIB-202 ${ }^{\mathrm{TM}}$ ), MOLT-4 human T lymphoblast cell line (ATCC ${ }^{\circ}$ CRL- $1582^{\mathrm{TM}}$ ), U937 histiocytic lymphoma cell line (ATCC ${ }^{\circledR}$ CRL1593.2 ${ }^{\mathrm{TM}}$ ) and $\mathrm{K}-562$ human myelogenous leukemia cell line $\left(\right.$ ATCC $^{\oplus} \mathrm{CCL}^{2433^{\mathrm{TM}}}$ ) were maintained at a logarithmic growth phase in complete RPMI (RPMI1640 supplemented with $10 \%$ heat-inactivated fetal calf serum (FCS), $2 \mathrm{mM}$ L-glutamine, 50 units $/ \mathrm{ml}$ penicillin, $50 \mu \mathrm{g} / \mathrm{ml}$ streptomycin (all from Biological Industries, Beit Haemek, Israel), and $0.5 \mu \mathrm{M} \beta$-mercaptoethanol (Sigma)). COS-7 epithelial kidney cells, which are derived from an African green monkey and express the Simian Vacuolating Virus 40 (SV40) TAg (ATCC ${ }^{\oplus}$ CRL-1651 $^{\text {TM }}$ ), A-549 human lung carcinoma cell line $\left(\mathrm{ATCC}^{\circ} \mathrm{CCL}^{\mathrm{C}}-185^{\mathrm{TM}}\right.$ ), MDA-MB 453 human breast metastatic carcinoma cell line (ATCC $^{\circ}$ HTB- $^{-131^{\mathrm{TM}}}$ ), MCF-7 human breast adenocarcinoma cell line (ATCC ${ }^{\circ}$ HTB-22 $^{\text {TM }}$ ) and HEK-293T human embryonic kidney cells, which express the SV40 Tag, were maintained in complete Dulbecco's modified Eagle medium (DMEM; supplemented with 10\% heatinactivated FCS, $2 \mathrm{mM}$ L-glutamine, 50 units $/ \mathrm{ml}$ penicillin, $50 \mu \mathrm{g} / \mathrm{ml}$ streptomycin and $0.5 \mu \mathrm{M} \beta$-mercaptoethanol).

\section{Transient cell transfection}

COS-7 cells that were maintained at a logarithmic growth phase were collected by trypsin treatment, washed in supplement-free DMEM, resuspended at $2 \times 10^{6}$ cells/ $\mathrm{ml}$ in $5 \mathrm{ml}$ DMEM with 10\% FCS and cultured in Cellstar tissue culture flasks (Greiner Bio-One 690170). Within $24 \mathrm{~h}$ of plating, adherent cells were transfected with the indicated DNA ( $5 \mu \mathrm{g} /$ group) using polyethylenimine (PEI, Polysciences Inc.) at a ratio of 3:1 (PEI: total DNA) and analyzed after two additional days in culture.

\section{Preparation of cell lysates}

Cells were resuspended in a lysis buffer $(25 \mathrm{mM}$ Tris$\mathrm{HCl}, \mathrm{pH}$ 7.5, $150 \mathrm{mM} \mathrm{NaCl}, 5 \mathrm{mM}$ EDTA, $1 \mathrm{mM} \mathrm{Na}_{3} \mathrm{VO}_{4}$, $50 \mathrm{mM} \mathrm{NaF}, 10 \mu \mathrm{g} / \mathrm{ml}$ each of leupeptin and aprotinin, $2 \mathrm{mM}$ AEBSF and $1 \%$ Triton X-100) followed by $30 \mathrm{~min}$ incubation on ice and centrifugation at $13,000 \mathrm{rpm}$ for $30 \mathrm{~min}$ at $4{ }^{\circ} \mathrm{C}$. Equal volumes of $2 \times$ sodium dodecyl sulfate (SDS) sample buffer were added to whole cell lysates (WCL) followed by vortexing, boiling for $5 \mathrm{~min}$, and sample fractionation by SDS polyacrylamide gel electrophoresis (PAGE).

\section{Electrophoresis and immunoblotting}

Samples containing whole cell lysates, chromatin lysates, or $\mathrm{Ab}$ immunoprecipitates were resolved by electrophoresis on 10 or $12.5 \%$ polyacrylamide gels using Bio-Rad Mini-PROTEAN II cells. Gel proteins were electroblotted onto nitrocellulose membranes (Schleicher and Schuell) at $100 \mathrm{~V}$ for $1 \mathrm{~h}$, using BioRad Mini TransBlot transfer cells. After $1 \mathrm{~h}$ of membrane blocking with $3 \% \mathrm{BSA}$ in TBS at $37^{\circ} \mathrm{C}$, the membranes were incubated with the indicated primary Abs followed by extensive washings in TBS and incubation with HRP-conjugated goat anti-mouse or goat anti-rabbit IgG Abs. Immunoreactive protein bands were visualized using the enhanced chemiluminescence (ECL) detection system followed by autoradiography.

\section{Chromatin immunoprecipitation and real-time qPCR}

Chromatin immunoprecipitation (ChIP) was performed according to a published protocol ${ }^{52}$, with few modifications. Cells were crosslinked using $1 \%$ formaldehyde (Sigma-Aldrich, Israel) for $10 \mathrm{~min}$ at room temperature, washed twice in cold PBS, and then lysed by resuspension in $1 \mathrm{ml}$ lysis buffer $(20 \mathrm{mM}$ Tris- $\mathrm{HCl}, \mathrm{pH} 8.0,85 \mathrm{mM} \mathrm{KCl}$, $0.5 \% \mathrm{NP}-40$ and $1 \%$ protease inhibitor cocktail) for $10 \mathrm{~min}$ on ice. Nuclear pellets obtained by centrifugation $(5 \mathrm{~min}$, $5000 \mathrm{rpm}, 4^{\circ} \mathrm{C}$ ) were resuspended in $200 \mu \mathrm{l}$ nuclei lysis buffer (50 mM Tris-HCl, pH 8.0, 10 mM EDTA, 1\% SDS and $1 \%$ protease inhibitor cocktail) for $10 \mathrm{~min}$ on ice, and samples were sheared by 5 rounds of 6 cycles of $30 \mathrm{~s}$ on/ $30 \mathrm{~s}$ off at a high-power setting of the Bioruptor sonicator (Diagenode), to yield input DNA enriched fragment sized 200-1000 bp.

The dimension of the chromatin fragments was determined empirically by agarose gel electrophoresis and ethidium bromide staining. Samples were spun down $\left(20 \mathrm{~min}, 13,000 \mathrm{rpm}, 4{ }^{\circ} \mathrm{C}\right.$ ) and the soluble chromatin fractions were collected, diluted (1:5) in a dilution buffer (0.01\% SDS, $1.1 \%$ Triton X-100, $1.1 \mathrm{mM}$ EDTA, $20 \mathrm{mM}$ Tris- $\mathrm{HCl}, \mathrm{pH} 8.0$, and $167 \mathrm{mM} \mathrm{NaCl}$ ) and incubated with protein G-sepharose beads for a preclearing step. Precleared lysates were incubated with bead-mobilized specific $\mathrm{Ab}$ or nonspecific isotype control $\mathrm{Ab}$ and kept 
overnight on a rotator at $4{ }^{\circ} \mathrm{C}$. The following day, the beads were sequentially washed with buffer-1, buffer-2, and buffer-3, and twice with buffer- 4 .

Buffers used are listed as below:

Buffer-1: 0.1\% SDS, 1\% Triton X-100, 2 mM EDTA, $20 \mathrm{mM}$ Tris-Cl, pH 8.0, $150 \mathrm{mM} \mathrm{NaCl}$ in $\mathrm{ddH}_{2} \mathrm{O}$

Buffer-2: $0.1 \%$ SDS, $1 \%$ Triton X-100, 2 mM EDTA, $20 \mathrm{mM}$ Tris-Cl, pH 8.0, $500 \mathrm{mM} \mathrm{NaCl}$ in $\mathrm{ddH}_{2} \mathrm{O}$

Buffer-3: $0.25 \mathrm{M} \mathrm{LiCl}, 1 \% \mathrm{NP} 40,1 \%$ deoxycholate, $1 \mathrm{mM}$ EDTA, $20 \mathrm{mM}$ Tris-Cl, pH 8.0 in $\mathrm{ddH}_{2} \mathrm{O}$

Buffer-4: $10 \mathrm{mM}$ Tris-HCl, 8.0 in $1 \mathrm{mM}$ EDTA

Precipitated DNA fragments were eluted from the protein $\mathrm{G}$ sepharose beads using elution buffer (1\% SDS, $50 \mathrm{mM} \mathrm{NaHCO}, 140 \mathrm{mM} \mathrm{NaCl}, 10 \mathrm{mM}$ Tris- $\mathrm{HCl}, \mathrm{pH}$ 8.0, 1 mM EDTA). Subsequently, eluted DNA was treated with RNAase $(0.2 \mu \mathrm{g} / \mu \mathrm{l})$ at $37^{\circ} \mathrm{C}$ for $1 \mathrm{~h}$, followed by incubation with proteinase $\mathrm{K}$ at $65^{\circ} \mathrm{C}$ for $5 \mathrm{~h}$. The DNA was then purified using PCR clean-up kit (MachereyNagel kit) and amplified by real-time qPCR (40 cycles (Cycling stage: $15 \mathrm{~s} 95^{\circ} \mathrm{C} / 60 \mathrm{~s} 60^{\circ} \mathrm{C}$; melt curve stage: $15 \mathrm{~s}$ $95^{\circ} \mathrm{C} / 60$ s $60^{\circ} \mathrm{C} / 30$ s $95^{\circ} \mathrm{C} / 60$ s $\left.60^{\circ} \mathrm{C}\right)$ ) with primers specific for the indicated target genes. The forward and reverse primer sequences used in the RT-PCR experiments were as follows:

CCND2 ChIP-qPCR forward primer: TAGGATCC GTTTTGAAGAAGCC, reverse CATTCTGTAGGTGTA GCACGCC $^{53}$; HOXA2 ChIP-qPCR forward primer: AGGAAAGATTTTGGTTGGGAAG, reverse AAAAAG AGGGAAAGGGACAGAC ${ }^{54}$, ATF3 ChIP-qPCR forward primer: CTACAGTCACCTTGCGGTGC, reverse GAG GCTGGGAAGGGTAATGG ${ }^{55}$; CDC6 ChIP-qPCR forward primer: GATTCCCTCCCCCGTTCA, reverse primer: CAAT GAGAGAGCCCCAAGTCTT ${ }^{53}$; GAPDH ChIP-qPCR forward primer: TACTAGCGGTTTTACGGG CG, reverse TCGAA CAGGAGGAGCAGAGAGCGA. Commercial primers for the $\alpha$-Satellite were obtained from Cell signaling (\#4486).

ChIP qPCR quantification was carried out as the percentage of the input chromatin. The relative enrichment was calculated by dividing the DNA amount obtained using the specific Ab, by the DNA amount obtained using the isotype control, for any of the targeted primers ${ }^{54}$.

$\%$ of Input $=100 \times 2^{\text {(adjusted input-Ct (IP)) }}$; where adjusted input $=$ raw input $\mathrm{Ct}-\log 2$ of dilution factor.

Average results were obtained from two independent ChIP experiments that were analyzed by three independent PCRs $(N=6$ wells $)$.

\section{Protein-protein chromatin immunoprecipitation}

Protein-protein ChIP was performed according to a published protocol with few modifications ${ }^{56}$. Cell crosslinking and chromatin lysates were prepared as described in the 'chromatin immunoprecipitation and realtime qPCR' section. The soluble chromatin fraction was diluted (1:5) in dilution buffer (0.01\% SDS, $1.1 \%$ Triton
X 100, 1.1 mM EDTA, 20 mM Tris-HCl, pH 8, 167 mM $\mathrm{NaCl}$ ) and incubated with protein G-sepharose beads for a preclearing step. Precleared lysates were incubated with bead-mobilized specific or nonspecific isotype control $\mathrm{Ab}$ and kept overnight on a rotator at $4{ }^{\circ} \mathrm{C}$. The following day, beads were washed once using buffer-1, buffer-2, buffer-3 and twice with buffer-4, as indicated previously. After the last wash, $180 \mu$ of sample buffer were added to the pellet and samples were kept at $95^{\circ} \mathrm{C}$ for $30 \mathrm{~min}$. The samples were then centrifuged and the supernatants $(20 \mu \mathrm{l})$ were resolved by SDS-PAGE and analyzed by immunoblot.

\section{Cell staining and confocal microscopy}

Cells $\left(1.5 \times 10^{6}\right)$ were seeded on poly-L-lysine-coated 8 well $\mu$-slide (ibidi Ltd.) and deposited on the slides by centrifugation at $1200 \mathrm{rpm}$ for $5 \mathrm{~min}$. Cells were fixed in $4 \%$ paraformaldehyde/PBS for $15 \mathrm{~min}$ at room temperature, permeabilized with PBS/0.2\% Triton X-100 for $5 \mathrm{~min}$ and incubated with mouse anti-PICOT mAbs and rabbit anti-EED polyclonal Abs in a blocking buffer (PBS containing $1 \% \mathrm{BSA}$ ) for $1 \mathrm{~h}$ at room temperature. After three washes in PBS, the cells were incubated with Cy3conjugated goat anti-mouse Ig Abs (1:500), Cy5conjugated goat anti-rabbit Ig Abs (1:500), and DAPI $(1 \mu \mathrm{g} / \mathrm{ml})$ in PBS for $1 \mathrm{~h}$ at room temperature in the dark. Confocal microscope analysis was performed using the Olympus FluoView ${ }^{\circledast}$ FV1000 laser scanning confocal microscope.

\section{RNA extraction and quantitative PCR}

For quantitative PCR analysis, RNA was extracted from Jurkat and Jurkat-derived sublines using an RNA isolation kit (YRB100, RBC Bioscience Corp.) according to the manufacturer's instructions. For cDNA synthesis, $1 \mu \mathrm{g}$ of RNA was reverse transcribed (RT) with the Bio-RT first strand cDNA synthesis kit (Bio-Lab) using random hexamer primers. The reaction was carried out for $60 \mathrm{~min}$ at $37^{\circ} \mathrm{C}$ and terminated by incubation at $75^{\circ} \mathrm{C}$ for $15 \mathrm{~min}$. Real-time (RT)-PCR was performed on the ABI PRISM ${ }^{\mathrm{sx}}$ 7500 (Applied Biosystems ${ }^{\mathrm{su}}$, Foster City, CA), using ABsolute qPCR SYBR Green kit (ABgene Inc., Portsmouth, NH) according to the manufacturer's instructions. The RT-PCR reaction contained $50 \mathrm{ng}$ of cDNA product and $500 \mathrm{~nm}$ of each of the primers. Primers for each gene studied were designed with overlapping exon boundaries, using the NCBI-BLAST software, and obtained from Sigma-Aldrich, Israel. All primers were designed to have $<150 \mathrm{bp}$, to ensure high amplification efficiency. The forward and reverse primer sequences used for the RT-PCR amplification were as follows: $C C N D 2$ forward primer: GGACATCCAAC CCTACATGC, reverse CGCACTTCTGTTCCTCACAG; SDHA forward primer: ACAACTGGAGGTGGCATTT CTA, reverse TAATTTTCTAGCTCGACCACGG. 
All experiments were performed in triplicates to ensure reproducibility. The cycling conditions included a polymerase activation step at $95^{\circ} \mathrm{C}$ for $10 \mathrm{~min}$, followed by 40 cycles of $95^{\circ} \mathrm{C}$ for $15 \mathrm{~s}$ and $60^{\circ} \mathrm{C}$ for $60 \mathrm{~s}$. After PCR amplification, the specificity of the PCR was confirmed by melting temperature determination of the PCR product. Amplification efficiencies were verified by serial dilution standard curve fit. Relative quantification was determined by the ${ }^{\Delta \Delta} \mathrm{Ct}$ method ${ }^{57}$, standardized against succinate dehydrogenase (SDHA), as a reference gene, and expressed as fold change.

Tumor tissue and normal adjacent tissue data collections

Cancer patients' clinical outcome and RNA expression data from tumor tissues and normal adjacent tissues were downloaded from the Cancer Genome Atlas (TCGA) data portal at the ISB cancer genomics cloud (https://isb-cgc. appspot.com/data) ${ }^{58}$ on July 15,2018 . For gene expression data, we selected the level-3 RNA-Seq-V0 data (Illumina GA or HiSeq sequencing platforms). RNA-Seq expression level read-counts were normalized, using FPKM values (Fragments per Kilobase of transcript per Million mapped reads), as provided in the downloaded files. Additional information regarding the data and analysis methods used in the processing of TCGA data can be found at https:// docs.gdc.cancer.gov/Data/Bioinformatics_Pipelines/ Expression_mRNA_Pipeline.

\section{Patients' survival analysis}

For every gene analyzed, patients were classified according to the high or low expression of the gene of interest. The classification was performed by calculating a mean cut of point using the MaxStat function (which is based on the maximally selected rank statistics) ${ }^{59}$, as available in the R package survminer (https://cran.rstudio. com/web/packages/survminer/index.html). For plotting the survival of the low and high expression patient groups the Kaplan-Meier estimator ${ }^{60}$ was used.

\section{PICOT-CCND2 correlation analysis}

PICOT and CCND2 mRNA expression levels in solid human cancers were compared using the TCGA dataset and the Pearson correlation. Outlier samples in total population were identified using quantiles, and samples from the upper and lower 0.1 quantiles were removed from the actual analysis. To analyze for potential correlation between PICOT and CCND2 expression in TALL, we searched the Gene Expression Omnibus (GEO) repository and searched for T-ALL data series. The dataset selected (GSE62156) had 64 sample, which were downloaded and processed using an R script inspired by the GEO2R option. Probe set 207506_at was used for PICOT and 200951_s_at was used for CCND2.

\section{Statistical evaluation}

Statistical evaluation for sample comparison was carried out using the Prism5 software (GraphPad, La Jolla/CA, USA). Statistical tests used are indicated in the respective figure legends.

Statistical evaluation for TCGA datasets was carried out using R, version 3.4.1 (http://www.r-project.org). Patients' clinical outcome data were analyzed using the survival package and plotted as Kaplan-Meier survival curves.

Unless specified otherwise, $p$ values smaller than 0.05 were considered significant. An exact $p$ value was calculated where applicable.

\section{Acknowledgements}

This research was supported by the USA-Israel Binational Science Foundation, the Israel Science Foundation administered by the Israel Academy of Science and Humanities, and a donation by Mr. Martin Kolinsky. N.I. holds the Joseph H. Krupp Chair in Cancer Immunobiology. We thank Dieter Adam for the gift of reagents, Ariel Ohayon for the preparation of PICOT-deficient Jurkat T cell sublines, Margalit Krup for technical assistance, and Caroline Simon and Judith Isakov for editorial assistance.

Conflict of interest

The authors declare that they have no conflict of interest.

\section{Publisher's note}

Springer Nature remains neutral with regard to jurisdictional claims in published maps and institutional affiliations.

Supplementary Information accompanies this paper at (https://doi.org/ 10.1038/s41419-019-1935-0).

Received: 7 February 2019 Revised: 8 August 2019 Accepted: 27 August 2019

Published online: 17 September 2019

References

1. Babichev, et al. The protein kinase C (PKC) theta-interacting proein, PICOT, undergoes tyrosine phosphorylation in response to hydrogen peroxide. In Proceedings of the 2nd Congress of the Federation of Immunological Societies of Asia-Oceania (FIMSA 2000) Vol. 495 (eds. Sirisinha, S., Chaiyaroj, S. C. \& Tapchaisri, P.) 11-16 (Monduzzi Editore, Bologna, 2000).

2. Babichev, Y., Witte, S., Altman, A. \& Isakov, N. in The Human PICOT Protein Possesses A Thioredoxin-like Homology Domain and A Tandem Repeat of A Novel Domain Which is Highly Conserved during Evolution. Vol. 318 (IOS Press, $\mathrm{OHM}, 2001)$.

3. Isakov, N., Witte, S. \& Altman, A. PICOT-HD: a highly conserved protein domain that is often associated with thioredoxin and glutaredoxin modules. Trends Biochem. Sci. 25, 537-539 (2000).

4. Cheng, N. H. et al. A mammalian monothiol glutaredoxin, Grx3, is critical for cell cycle progression during embryogenesis. FEBS J. 278, 2525-2539 (2011).

5. Cha, H. et al. PICOT is a critical regulator of cardiac hypertrophy and cardiomyocyte contractility. J. Mol. Cell Cardiol. 45, 796-803 (2008).

6. Babichev, Y. \& Isakov, N. Tyrosine phosphorylation of PICOT and its translocation to the nucleus in response of human T cells to oxidative stress. Adv. Exp. Med. Biol. 495, 41-45 (2001).

7. Ohayon, A., Babichev, Y., Galperin, M., Altman, A. \& Isakov, N. Widespread expression of PICOT in mouse and human tissues with predominant localization to epithelium. J. Histochem. Cytochem. 58, 799-806 (2010).

8. Jeong, D. et al. PICOT inhibits cardiac hypertrophy and enhances ventricular function and cardiomyocyte contractility. Circ. Res. 99, 307-314 (2006). 
9. Haunhorst, P., Berndt, C., Eitner, S., Godoy, J. R. \& Lillig, C. H. Characterization of the human monothiol glutaredoxin 3 (PICOT) as iron-sulfur protein. Biochem. Biophys. Res. Commun. 394, 372-376 (2010).

10. Haunhorst, P. et al. Crucial function of vertebrate glutaredoxin 3 (PICOT) in iron homeostasis and hemoglobin maturation. Mol. Biol. Cell 24, 1895-1903 (2013).

11. Ohayon, A. et al. Hodgkin's lymphoma cells exhibit high expression levels of the PICOT protein. J. Immunotoxicol. 7, 8-14 (2010).

12. Cha, M. K. \& Kim, I. H. Preferential overexpression of glutaredoxin3 in human colon and lung carcinoma. Cancer Epidemiol. 33, 281-287 (2009).

13. Pandya, P. et al. PICOT binding to the polycomb group protein, EED, alters H3K27 methylation at the MYT1 PRC2 target gene. Biochem. Biophys. Res. Commun. 509, 469-475 (2019)

14. Whitcomb, S. J., Basu, A., Allis, C. D. \& Bernstein, E. Polycomb Group proteins: an evolutionary perspective. Trends Genet. 23, 494-502 (2007).

15. Margueron, R. \& Reinberg, D. The Polycomb complex PRC2 and its mark in life. Nature 469, 343-349 (2011).

16. Pirrotta, V. PCG complexes and chromatin silencing. Curr. Opin. Genet. Dev. 7, 249-258 (1997).

17. Cao, Q. et al. The central role of EED in the orchestration of polycomb group complexes. Nat. Commun. 5, 3127 (2014).

18. Bolte, S. \& Cordelieres, F. P. A guided tour into subcellular colocalization analysis in light microscopy. J. Microsc. 224, 213-232 (2006).

19. Philipp, S. et al. The Polycomb group protein EED couples TNF receptor 1 to neutral sphingomyelinase. Proc. Natl Acad. Sci. USA 107, 1112-1117 (2010).

20. Lanzuolo, C. \& Orlando, V. Memories from the polycomb group proteins. Annu. Rev. Genet. 46, 561-589 (2012).

21. Kuzmichev, A., Jenuwein, T., Tempst, P. \& Reinberg, D. Different EZH2containing complexes target methylation of histone $\mathrm{H} 1$ or nucleosomal histone H3. Mol. Cell 14, 183-193 (2004).

22. Margueron, R. et al. Role of the polycomb protein EED in the propagation of repressive histone marks. Nature 461, 762-767 (2009).

23. Montgomery, N. D. et al. The murine polycomb group protein Eed is required for global histone H3 lysine-27 methylation. Curr. Biol. 15, 942-947 (2005).

24. Qu, Y. et al. Thioredoxin-like 2 regulates human cancer cell growth and metastasis via redox homeostasis and NF-kappaB signaling. J. Clin. Invest. 121, 212-225 (2011).

25. Witt, D. et al. Valproic acid inhibits the proliferation of cancer cells by reexpressing cyclin D2. Carcinogenesis 34, 1115-1124 (2013).

26. Jeong, D. et al. PICOT attenuates cardiac hypertrophy by disrupting calcineurin-NFAT signaling. Circ. Res. 102, 711-719 (2008).

27. Myant, K. \& Sansom, O. Efficient Wht mediated intestinal hyperproliferation requires the cyclin D2-CDK4/6 complex. Cell Div. 6, 3 (2011).

28. Sun, C. C. et al. The IncRNA PDIA3P Interacts with miR-185-5p to modulate oral squamous cell carcinoma progression by targeting cyclin D2. Mol. Ther. Nucleic Acids 9, 100-110 (2017).

29. Taieb, F., Chartrain, I., Chevalier, S., Haccard, O. \& Jessus, C. Cyclin D2 arrests Xenopus early embryonic cell cycles. Exp. Cell Res. 237, 338-346 (1997).

30. Sanulli, S. et al. Jarid 2 methylation via the PRC2 complex regulates H3K27me3 deposition during cell differentiation. Mol. Cell 57, 769-783 (2015).

31. Faust, C., Schumacher, A., Holdener, B. \& Magnuson, T. The eed mutation disrupts anterior mesoderm production in mice. Development 121, 273-285 (1995).

32. Wang, J., Mager, J., Schnedier, E. \& Magnuson, T. The mouse PCG gene eed is required for Hox gene repression and extraembryonic development. Mamm. Genome 13, 493-503 (2002).

33. Morin-Kensicki, E. M., Faust, C., LaMantia, C. \& Magnuson, T. Cell and tissue requirements for the gene eed during mouse gastrulation and organogenesis. Genesis 31, 142-146 (2001)

34. Margueron, R. et al. Ezh1 and Ezh2 maintain repressive chromatin through different mechanisms. Mol. Cell 32, 503-518 (2008).

35. Denisenko, O. N. \& Bomsztyk, K. The product of the murine homolog of the Drosophila extra sex combs gene displays transcriptional repressor activity. Mol. Cell Biol. 17, 4707-4717 (1997).

36. Ogawa, M., Hiraoka, Y. \& Aiso, S. The Polycomb-group protein ENX-2 interacts with ZAP-70. Immunol. Lett. 86, 57-61 (2003).
37. Denisenko, O., Shnyreva, M., Suzuki, H. \& Bomsztyk, K. Point mutations in the WD40 domain of Eed block its interaction with Ezh2. Mol. Cell Biol. 18, 5634-5642 (1998)

38. Su, I. H. et al. Polycomb group protein ezh2 controls actin polymerization and cell signaling. Cell 121, 425-436 (2005).

39. Pasini, D., Bracken, A. P., Jensen, M. R., Lazzerini Denchi, E. \& Helin, K. Suz12 is essential for mouse development and for EZH2 histone methyltransferase activity. EMBO J. 23, 4061-4071 (2004).

40. Montgomery, N. D., Yee, D., Montgomery, S. A. \& Magnuson, T. Molecular and functional mapping of EED motifs required for PRC2-dependent histone methylation. J. Mol. Biol. 374, 1145-1157 (2007).

41. Hauri, S. et al. A high-density map for navigating the human polycomb complexome. Cell Rep. 17, 583-595 (2016).

42. Smits, A. H., Jansen, P. W., Poser, I., Hyman, A. A. \& Vermeulen, M. Stoichiometry of chromatin-associated protein complexes revealed by label-free quantitative mass spectrometry-based proteomics. Nucleic Acids Res. 41, e28 (2013).

43. Conway, E. et al. A family of vertebrate-specific polycombs encoded by the LCOR/LCORL genes balance PRC2 subtype activities. Mol. Cell 70, 408-421 e408 (2018).

44. Virmani, A. et al. Aberrant methylation of the cyclin D2 promoter in primary small cell, nonsmall cell lung and breast cancers. Int. J. Cancer 107, 341-345 (2003).

45. Evron, E. et al. Loss of cyclin D2 expression in the majority of breast cancers is associated with promoter hypermethylation. Cancer Res. 61, 2782-2787 (2001).

46. Buckley, M. F. et al. Expression and amplification of cyclin genes in human breast cancer. Oncogene 8, 2127-2133 (1993).

47. Matsubayashi, $H$. et al. Methylation of cyclin D2 is observed frequently in pancreatic cancer but is also an age-related phenomenon in gastrointestinal tissues. Clin. Cancer Res. 9, 1446-1452 (2003).

48. Li, Y. L. et al. MiR-146a-5p inhibits cell proliferation and cell cycle progression in NSCLC cell lines by targeting CCND1 and CCND2. Oncotarget 7, 59287-59298 (2016).

49. Zhang, F., Zhu, Y., Fan, G. \& Hu, S. MicroRNA-2682-3p inhibits osteosarcoma cell proliferation by targeting CCND2, MMP8 and Myd88. Oncol. Lett. 16, 3359-3364 (2018).

50. Mo, X. et al. MicroRNA-610 suppresses the proliferation of human glioblastoma cells by repressing CCND2 and AKT3. Mol. Med. Rep. 13, 1961-1966 (2016).

51. Zhu, C. et al. miR-154 inhibits prostate cancer cell proliferation by targeting CCND2. Urol. Oncol. 32, 31 e39-16 (2014).

52. Ainbinder, E. et al. Mechanism of rapid transcriptional induction of tumor necrosis factor alpha-responsive genes by NF-kappaB. Mol. Cell Biol. 22, 6354-6362 (2002)

53. Van Dessel, N. et al. The phosphatase interactor NIPP1 regulates the occupancy of the histone methyltransferase EZH2 at Polycomb targets. Nucleic Acids Res. 38, 7500-7512 (2010).

54. Mahara, S. et al. HIFI-alpha activation underlies a functional switch in the paradoxical role of Ezh2/PRC2 in breast cancer. Proc. Natl Acad. Sci. USA 113 E3735-E3744 (2016)

55. Tiffen, J. C. et al. Targeting activating mutations of EZH2 leads to potent cell growth inhibition in human melanoma by derepression of tumor suppressor genes. Oncotarget 6, 27023-27036 (2015).

56. Nelson, J. D., Denisenko, O. \& Bomsztyk, K. Protocol for the fast chromatin immunoprecipitation (ChIP) method. Nat. Protoc. 1, 179-185 (2006).

57. Pfaffl, M. W. A new mathematical model for relative quantification in real-time RT-PCR. Nucleic Acids Res. 29, e45 (2001).

58. Reynolds, S. M. et al. The ISB cancer genomics cloud: a flexible cloud-based platform for cancer genomics research. Cancer Res. 77, e7-e10 (2017).

59. Lausen, B. \& Schumacher, M. J. Maximally selected rank statistics. Biometrics $\mathbf{4 8}$ 73-85 (1992)

60. Goel, M. K., Khanna, P. \& Kishore, J. Understanding survival analysis: KaplanMeier estimate. Int. J. Ayurveda Res. 1, 274-278 (2010). 